\title{
Hongos degradadores de la madera en el estado de Chihuahua, México
}

\author{
Wood decaying fungi from Chihuahua, Mexico
}

\author{
Raúl Díaz-Moreno ${ }^{1 *}$, Ricardo Valenzuela ${ }^{2}$, José Guadalupe Marmolejo ${ }^{3}$ y Elvira Aguirre-Acosta ${ }^{4}$ \\ ${ }^{1}$ Instituto de Silvicultura e Industria de la madera, Universidad Juárez del estado de Durango, Boulevard Durango 501, Cd. Universitaria, 34120, \\ Durango, Dgo. México. \\ ${ }^{2}$ Laboratorio de Micología, Departamento de Botánica, Escuela Nacional de Ciencias Biológicas, IPN, Apartado postal 260, Centro Operativo Naranjo, \\ Col. Santa María la Rivera, 02600 México, D. F., México \\ ${ }^{3}$ Facultad de Ciencias Forestales, Universidad Autónoma de Nuevo León, Apartado postal 41, 67700 Linares, Nuevo León, México \\ ${ }^{4}$ Colección de Hongos, Departamento de Botánica, Instituto de Biología, Universidad Nacional Autónoma de México, Apartado postal 70-233, 04510 \\ México, D. F., México. \\ *Correspondencia: r_diaz54@hotmail.com
}

\begin{abstract}
Resumen. Se presenta una lista de 83 especies de hongos principalmente poliporoides degradadores de la madera que habitan en bosques de pino y encino-pino; de las cuales, 37 son registros nuevos para el estado de Chihuahua. Las familias Polyporaceae e Hymenochaetaceae fueron las más representativas, y los géneros con mayor número de especies, Phellinus e Inonotus.

Palabras clave: Polyporaceae, Hymenochaetaceae, Chihuahua, México.

Abstract. This paper presents a list of 83 species, most of them polypore wood decaying fungi from pine forest and oak-pine forest. Thirty seven species are new records for the state of Chihuahua. The families Polyporaceae and Hymenochaetaceae were the most abundant, and the genera with the highest number of species were Phellinus and Inonotus.
\end{abstract}

Key words: Polyporaceae, Hymenochaetaceae, Chihuahua, Mexico.

\section{Introducción}

Florísticamente, la zona árida chihuahuense es única y muy importante en México por su alto grado de endemismos (Rzedowski, 1978). La flora del desierto chihuahuense se estima en 3500 especies, con un endemismo del 30\% (Johnston, 1943; LeSueur, 1945; Corell y Johnston, 1970). Por lo que se refiere a la micoflora de esta zona, Vovides et al. (1997) registran 6 especies que se encuentran en peligro de extinción por explotación : Agaricus augustus, Amanita caesarea, A. muscaria, Boletus edulis, Leccinum aurantiacum e Hygrophorus russula. Pérez Silva y Aguirre Acosta (1986) presentaron un lista de la micoflora de la sierra Tarahumara, en el estado de Chihuahua, con la que se amplió la distribución de los géneros ya conocidos en otras localidades del país. Laferrière y Gilbertson (1990 a, b; 1992) describieron 2 especies nuevas: Albatrellus mexicanus y Polyporus tenuiparies e hicieron un listado de macromicetes del estado registrando un total de 118 especies. Moreno-Fuentes et al. (1994) realizaron un trabajo

Recibido: 14 agosto 2007; aceptado: 03 junio 2008 taxonómico, específicamente en el municipio de Bocoyna, y mencionan 4 especies de la familia Hymenochaetaceae y 9 de la Polyporaceae; Quiñónez-Martínez (1999), registra para el estado 5 hongos destructores de la madera (Daedalea quercina, Ganoderma applanatum, Lenzites betulina, Phaeolus schweinitzii y Stereum complicatum).

\section{Materiales y métodos}

Área de estudio. El estado de Chihuahua se sitúa entre $\operatorname{los} 31^{\circ} 47^{\prime}-25^{\circ} 38^{\prime} \mathrm{N}$ y $103^{\circ} 18^{\prime}-109^{\circ} 07^{\prime} \mathrm{O}$. No obstante la diversidad de condiciones geológicas, topográficas y climáticas en el territorio chihuahuense, es posible identificar zonas cuyo paisaje general y características ecológicas tienden a ser homogéneas, o bien, conformar patrones típicos que permiten su identificación como grandes regiones naturales. En el estado se distinguen 3 grandes regiones que tanto por su paisaje como por su economía presentan patrones característicos: la sierra, al poniente, donde se encuentran las zonas boscosas y los climas más húmedos; la región de los valles centrales, 
en los que se asienta la mayor parte de la agricultura de temporal y la mayor concentración de la población rural, y el altiplano, desierto o mesa del norte, como se le conoce en diversas fuentes.

Debido a su accidentada orografía, Chihuahua goza de diversos climas. En la zona norte el clima es templado; la zona este es seca-desértica; el sur es semicálido; en el lado oeste impera el clima subhúmedo, que varía dependiendo de la altitud. La temperatura promedio anual es de $28{ }^{\circ} \mathrm{C}$, como máxima, y $10.9{ }^{\circ} \mathrm{C}$ como mínima (INEGI, 2001). Con $244938 \mathrm{~km}^{2}$, el estado de Chihuahua es el estado con mayor extensión en la República Mexicana; presenta 14 tipos de vegetación: bosque de pino, bosque de pinoencino, bosque de encino-pino, bosque de encino, bosque de tascate, selva baja caducifolia, matorral desértico micrófilo, matorral desértico micrófilo-ubinerme, matorral desértico micrófilo inerme, matorral desértico espinoso, matorral desértico rosetófilo, matorral con rosetófilos acaules, pastizal natural y vegetación halófila (FloresVillela y Gerez,1994). Durante los años de 1998 a 2003 se realizaron 7 salidas a campo recolectándose ejemplares en varias localidades y municipios del estado de Chihuahua. Cada ejemplar fue etiquetado con los datos de campo tales como localidad, fecha, sustrato, hábitat, nombre del recolector y número de colecta. Se procedió al secado del material, y una vez seco se guardó en cajas de cartón con su respectiva etiqueta, como lo recomienda Guzmán (1977). Todo el material recolectado por el primer autor se encuentra depositado en el herbario de la Facultad de Ciencias Forestales de la Universidad Juárez del estado de Durango (FCF-UJED); el resto, en el herbario de la Escuela Nacional de Ciencias Biológicas (ENCB-IPN).

Se revisaron las recolectas de hongos degradadores de la madera provenientes de Chihuahua que se encuentran depositados en los herbarios del Instituto de Biología (MEXU) y de la Facultad de Ciencias (FCME), ambos de la Universidad Nacional Autónoma de México, así como del herbario de Arizona (ARIZ). Entre otros, se tomaron datos de su hábitat y distribución. Los de localidades, presentados en la lista, fueron tomados de las etiquetas de herbario. Sin embargo, muchas carecían de la información o estaba incompleta, por lo que se recurrió a cartas topográficas del Catálogo de Integración General de Localidades (CIGELI) editadas por INEGI (2001) en las que se obtuvo el tipo de vegetación, la altitud y las coordenadas geográficas. Todo el material considerado en este trabajo proviene de 51 localidades pertenecientes a 14 municipios del estado, según se aprecia en la figura 1 y el Apéndice 2.

Para la descripción macroscópica de las especies se tomaron en cuenta las características de las siguientes estructuras del basidioma: píleo, estípite (cuando está presente) e himenóforo; se consideró tamaño de los poros

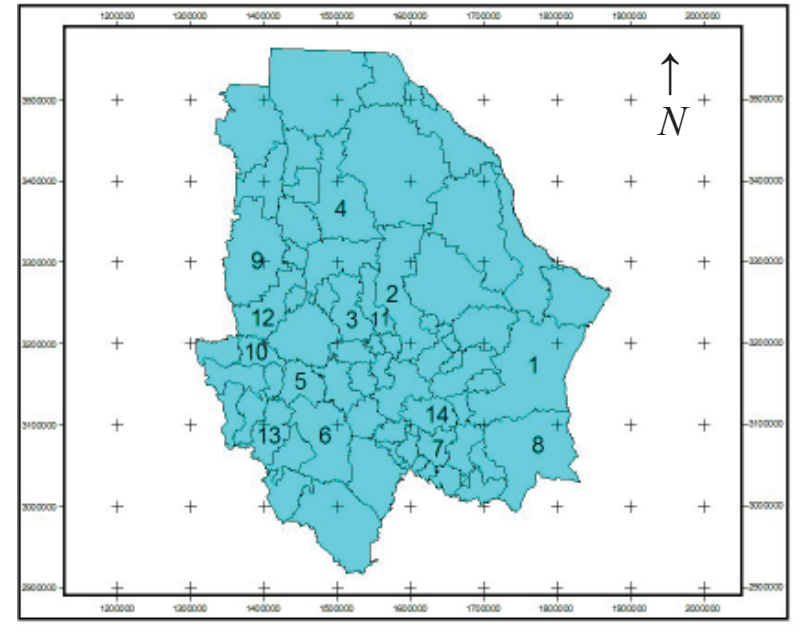

Figura 1. Municipios en el estado de Chihuahua donde se localizan los sitios de colecta. 1, Camargo; 2, Chihuahua; 3, Cuauhtemoc; 4, Buenaventura; 5, Bocoyna; 6, Guachochi; 7, Hidalgo del Parral; 8, Jiménez; 9, Madera; 10, Ocampo; 11, Riva Palacio; 12, Temosiche; 13, Urique; 14, Valle de Zaragoza.

por milímetro, color, disposición, forma, margen, tubos, estípite y contexto. La mayor parte de las fotografías se tomaron en el campo.

Para la identificación microscópica de las especies se hicieron preparaciones temporales con $\mathrm{KOH}$ al $5 \%$ y reactivo de Melzer, realizando cortes transversales y longitudinales de las diferentes partes del basidioma, tales como píleo, contexto, himenio y estípite; se tomaron los datos de importancia taxonómica, como tamaño, forma y color de las esporas, basidios, cistidios, setas e hifas; así también, se observó el tipo de sistema hifal y presencia de fíbulas. Para lo anterior se utilizaron las claves y la literatura especializada (Overholts, 1953; Pegler, 1964; Cunningham, 1965; Bondartsev,1971; Domanski, 1972; Domanski et al., 1973; Gilbertson, 1976; Ryvarden y Johansen, 1980; Gilbertson y Ryvarden, 1986, 1987, y Ryvarden y Gilbertson, 1993 a, b).

Para la validación de la sinonimia y nomenclatura de los géneros y especies estudiadas se consultó el Index fungorum.

\section{Resultados}

Mediante revisión macro y microscópica de los ejemplares recolectados, se determinaron 119 taxa, 83 en el nivel específico y 36 en el genérico. De estas especies, 46 habían sido previamente registradas y 37 son registros nuevos para el estado. Las familias Polyporaceae e Hymenochaetaceae fueron las más representativas con 26 y 
24 especies, respectivamente, y Phellinus con 11, Inonotus 9, Stereum 7, Polyporus y Trametes 5 y Gloeosporum 4, los géneros con mayor número de especies (Apéndice 1).

\section{Discusión}

El estado de Chihuahua es el estado más grande de la República Mexicana, pero no el más estudiado desde un punto de vista micológico, según se puede observar en los antecedentes, donde se menciona un total de 338 especies de macromicetes conocidos, de los que sólo se conocen 40 poliporoides, según Bandala et al. (1993). En el presente trabajo se registran por primera vez para el estado 37 especies de hongos de este grupo. El municipio que presentó un mayor número de sitios de colecta fue Guachochi con 20, seguido de Bocoyna con 10, por lo que se sugiere continuar con este tipo de estudios en estos sitios y conocer de manera más profunda su micobiota.

Es de esperarse que dada su orografía, lo cambiante de su clima y régimen de precipitación en el estado puedan encontrarse todavía un buen número de especies no descritas aún.

La importancia de estos hongos radica en que muchos son saprobios y contribuyen en los procesos de regeneración forestal y formación del suelo, y como parásitos activan el proceso de selección, formando individuos más resistentes.

Por lo anterior, se requiere continuar con el estudio micológico de esta zona que permita tener un conocimiento mejor de este grupo de hongos y poder realizar acciones de aplicación práctica con el recurso.

\section{Literatura citada}

Bandala, V. M., G. Guzmán y L. Montoya. 1993. Los hongos del grupo de los poliporáceos conocidos en México. Revista Mexicana de Micología, Facultad de Ciencias Forestales, Universidad Autónoma de Nuevo León, Linares. Reporte científico número especial 13: 1-55.

Bondartsev, A. S. 1971. The Polyporaceae of the European USSR and Caucasia. Traducción de U.S. Dept. Comerce, National Technical Informations Service, Springfield. Original en ruso, $1953.897 \mathrm{p}$.

Corell, D. S. y M. C. Johnston. 1970. Manual of the vascular plants of Texas. Texas Research Foundation, Renner. 1881 p.

Cunningham, G. H. 1965. Polyporaceae of New Zealand. New Zealand. Department Scientific Industrial Research. Bulletin. 164: 1-304

Domanski, S. 1972. Fungi Polyporaceae I (resupinate), Mucronoporaceae I (resupinate). Department of Agriculture and the National Science Fundation, Washington, D. C. 234 p.
Domanski, S., H. Orlos, y A. Skirgello. 1973. Basidiomycetes: Aphyllophorales: Polyporaceae pileate, Ganodermataceae, Bondarzewiaceae, Boletopsidaceae, Fistulinaceae. In Flora Polska (Grzyby) 3: 398.

Domànski, S., H. Ortos y A. Skirgietto. 1973. Fungi III. Polyporaceae II (Pileate), Mucroporaceae II (Pileate), Ganodermataceae, Bondarzewiaceae, Boletopsidaceae and Fistulinaceae. The U.S. Dep. of Agr. Natural Science Found. Washington, D.C. $234 \mathrm{p}$.

Flores-Villela, O. y P. Gerez. 1994. Biodiversidad y conservación en México: vertebrados, vegetación y uso del sueloComisión Nacional para el conocimiento y uso de la Biodiversidad y Universidad Nacional Autónoma de México, México, D.F. $439 \mathrm{p}$.

Gilbertson, R. L. 1976. The genus Inonotus (Aphyllophorales, Hymenochaetaceae) in Arizona. Memoires of the New York. Botanical Garden 28: 67-85.

Gilbertson, R. L. y L. Ryvarden. 1986. North American Polypores, vol.1 Abortiporus-Lindtneria. Fungiflora, Oslo. p. 433.

Gilbertson, R. L. y L. Ryvarden. 1987. North American Polypores, vol. 2 Megasporoporia-Wrightoporia. Fungiflora, Oslo. p. 434-885.

Guzmán, G. 1977. Identificación de los hongos comestibles, venenosos, alucinantes y destructores de la madera. Limusa, México, D. F. 452 p.

Index fungorum. www.indexfungorum.org/Names/Names.asp 12 febrero 2009.

INEGI. 2001. Catálogo de Integración General de Localidades. (CIGELI 2001) Instituto Nacional de Estadística Geografía e Informática, Aguascalientes, Aguascalientes. CD.

INEGI. 2001. Cuaderno de información del estado de Chihuahua. Instituto Nacional de Estadística Geografía e Informática, Aguascalientes, Aguascalientes. 568 p.

Johnston, L. M. 1943. Plants of Coahuila, eastern Chihuahua and adjoining Zacatecas and Durango. II. Journal of the Arnold Arboretum 24: 371-421.

Laferrière, J. E. y R. L. Gilbertson. 1990 a. A new species of Polyporus (Aphyllophorales: Polyporaceae) from Mexico. Mycotaxon 37: 331-333.

Laferrière, J. L. y R. L. Gilbertson. 1990.b A new species of Albatrellus (Aphyllophorales: Albatrellaceae) from Mexico. Mycotaxon 37: 183-186.

Laferrière, J. E. y R. L. Gilbertson. 1992. Fungi of Nabogame, Chihuahua, Mexico. Mycotaxon 44: 73-87.

LeSueur, H. 1945. The ecology of the vegetation of Chihuahua, Mexico, north of parallel 28. University Texas Publisher 4521, Austin. 92 p.

Moreno-Fuentes A., E. Aguirre-Acosta, M. Villegas y J. Cifuentes. 1994. Estudio fungístico de los macromicetos en el municipio de Bocoyna, Chihuahua, México. Revista Mexicana Micología 10: 63-76.

Overholts, L. O. 1953. The Polyporaceae of the United States, Alaska and Canada. University of Michigan Press, Ann Arbor $466 \mathrm{p}$.

Pegler, D. N. 1964. A survey of the genus Inonotus (Polyporaceae). Transactions British Mycolological Society 47: 175-195.

Pérez-Silva E. y E. Aguirre-Acosta. 1986. Flora micológica del estado de Chihuahua, México. I. Anales del Instituto 
Biología Universidad Nacional Autónoma de México, Serie Botánica 57:17-32.

Quiñónez-Martínez, M. 1999. Taxonomía, ecología y distribución de hongos macromicetos de Bosque Modelo, Chihuahua. Tesis, maestría Universidad Autónoma de Chihuahua, Chihuahua. 285 p.

Ryvarden, L. y I. Johansen. 1980. A preliminary polypore flora of east Africa. Fungiflora, Oslo. 636 p.

Ryvarden, L. y R .L. Gilbertson. 1993a. European Polypores, part. 1. Fungiflora, Oslo. p. 1-387.

Ryvarden, L. y R. L. Gilbertson. 1993b. European polypores, part. 2. Fungiflora, Oslo. p. 388-743.

Rzedowzki, J. 1978. La vegetación de México. Limusa, México, D. F. 395 p.

Vovides, A. P., V. Luna y G. Medina. 1997. Relación de algunas plantas y hongos mexicanos raros, amenazados o en peligro de extinción y sugerencias para su conservación. Acta Botanica Mexicana 39: 1-42.

Apéndice 1. Lista de especies de hongos degradadores de la madera en el estado de Chihuahua, México.

*nuevos registros para el estado. Loc., localidad; véase la Lista de localidades por municipio (Apéndice 2).

\section{Schizophyllaceae}

\section{Orden AGARICALES}

*Schizophyllum commune $\mathrm{Fr}$

Observ. Mycol. 1:330, 1815

Material estudiado. Loc. 18, 12 agosto, 1998, Díaz Moreno 590. Loc. 23, 30 agosto, 1980, E. Pérez Silva 116286 (MEXU), 30 agosto, 1980, E. Pérez Silva 16393 (MEXU), Loc. 36, 13 junio, 1915, E. O. Matthews s/n (ARIZ), Loc. 37, 14 abril, 1915, E. O. Matthews s/n (ARIZ).

\section{Coniophoraceae}

\section{Orden BOLETALES}

\section{Coniophora inflata Burt}

Ann. Mo. Bot. Gdn. 4:247, 1917

Material estudiado. Loc. 42, 22 agosto, 1915, E. O. Matthews 290504 (ARIZ).

\section{Hymenochaetaceae \\ Orden HYMENOCHAETALES \\ *Coltricia cinnamomea (Jacq.) Murr.

$$
\text { Bull. Torr. Bot. Club. 31(6):343, } 1904
$$

Material estudiado. Loc. 4, 15 marzo, 1981, P. Domínguez s/n (ENCB), Loc. 8, 20 septiembre, 1992, Moreno Fuentes 5625 (FCME), Loc. 16, 11 agosto, 1998, Díaz Moreno 570 (FCFUJED).

Coltricia perennis (Fr.) Murr.

J. Mycol. 9:91, 1903

Material estudiado. Loc. 13, 9 agosto, 2003, Díaz Moreno 749 Loc. 16, 29 agosto, 1980, E. Pérez Silva 16502 (MEXU), Loc. 18, 26 septiembre, 1996, Díaz Moreno 514, Loc. 25, 29 agosto, 1980, E. Pérez Silva 16393 (MEXU), Loc. 42, 27 septiembre, 1978, E. Pérez Silva 12717 (MEXU), 27 septiembre, 1980, E. Pérez Silva 16295 (MEXU).

Hymenochaete sallei B. et C. J. Linn. Soc. Bot. 10(46): 333, 1868

Material estudiado. Loc. 39, 8 septiembre, 1998, Díaz Moreno 548 (FCF-UJED).

*Hymenochaete tabacina (Sowerby: Fr.) Lév. Ann. Sci. Nat. Bot. III 5: 145, 1846

Material estudiado. Loc. 39, 8 septiembre, 1998, Díaz Moreno 553 (FCF-UJED).

*Inonotus circinatus (Fr.) Gilbn. Chung-kuo Ti Chen-Chun: 107, 1964 Material estudiado. Loc. 4, 3 mayo, 1981, P. Domínguez s/n (ENCB).
*Inonotus cuticularis (Bull.) Karst.

Meddn. Soc. Fauna Flora. fenn. 5: 39, 1880

Material estudiado. Loc. 36, 12 diciembre, 1944, E. O. Matthews 208021 (ARIZ), 27 enero, 1914, E. O. Matthews 207178 (ARIZ)

*Inonotus dryadeus (Pers.) Murr. North Am. Flora 9(2): 86, 1908

Material estudiado. Loc. 17, 11 agosto, 1998, Díaz Moreno 585 (FCF-UJED).

*Inonotus fulvomelleus Murr. (Fig. 2) North. Am. Flora. 9(2): 87, 1908

Material estudiado. Loc. 33, 20 abril, 1999, Cebrián Tovar 33B (ENCB).

Inonotus farlowii (Lloyd) Gilbn. Mem. N.Y. Bot. Gdn. 28: 77, 1976

Material estudiado. Loc. 2, 27 enero, 1915, E. O. Matthews s/n (ARIZ), Loc. 33, 20 abril, 1999, Cebrián Tovar 33-B (ENCB).

Loc. 36, 12 diciembre, 1914, E. O. Matthews s/n (ARIZ).

*Inonotus hispidus (Bull.) Karst.

Meddn Soc. Fauna Flora fenn. 5:39, 1880

Material estudiado. Loc. 4, 8 septiembre, 1992, Moreno

Fuentes F-III-1 (FCME), Loc. 34, 20 abril, 1999, Cebrián Tovar 32 (ENCB), Loc. 35, 19 abril, 1999, Cebrián Tovar 29 (ENCB).

*Inonotus jamaiciensis Murr. Bull. Torr. Bot. Club 31:597, 1904

Material estudiado. Loc. 6, 10 septiembre, 1998, Díaz Moreno 567-B, Loc. 17, 11 septiembre, 1998, Díaz Moreno 587, Loc. 39, 8 septiembre, 1998, Díaz Moreno 552 (FCF-UJED). Inonotus munzii (Lloyd) Gilbn. Southwestern Nat. 14: 125, 1969

Material estudiado. Loc. 2, 14 diciembre, 1942, E. O. Matthews s/n (ARIZ). Loc. 3, 12 diciembre, 1914, E. O. Matthews 207178 (ARIZ), Loc. 14, 13 abril, 1942, E. O. Matthews 213218

(ARIZ), Loc. 36, 12 diciembre, 1914, E. O. Matthews 213218

(ARIZ), 12 diciembre, 1914, E. O. Matthews 213217 (ARIZ),

14 diciembre, 1941, E. O. Matthews 213220 (ARIZ), Loc. 37, 13 abril, 1942, E. O. Matthews 213207 (ARIZ).

Inonotus tomentosus (Fr.) Teng Fungi of China p. 761, 1964

Material estudiado. Loc. 15, 10 noviembre, 1989, Méndez Montiel s/n (ENCB).

*Phellinus arctostaphyli (Long) Niemala (Fig. 3) Ann. Bot. Fenn. 12: 120, 1975

Material estudiado. Loc. 6, 10 septiembre, 1998, Díaz Moreno 567, Loc. 22, 26 agosto, 1980, E. Pérez Silva 16393 (MEXU), 
Loc. 28, 27 julio, 1981, J. C. Reyes 19915 (ENCB).

*Phellinus badius (Berk.) Cunn. 273, 1965

Bull. N. Z. Dep. Sci. Industr. Res. Pl. Dis. Div. 164:

Material Estudiado. Loc. 2, 20 diciembre, 1915, E. O.

Matthews 228527 (ARIZ), Loc. 6, 18 septiembre, 1992,

Moreno-Fuentes 5673 (FCME). Loc. 36, 14 diciembre, 1914,

E. O. Matthews s/n (ARIZ), 13 marzo, 1839, E. O. Matthews

228527 (ARIZ), 20 diciembre, 1914, E. O. Matthews 228533

(ARIZ).

Phellinus gilvus (Schw.) Pat.

Essai Tax. Hymenomyc.: 82, 1900

Material estudiado. Loc. 2, 13 abril, 1942, Hartmann 209477

(ARIZ), Loc. 14, 13 abril, 1942, E. O. Matthews 209455

(ARIZ), Loc. 43, 6 agosto, 1987, Laferrière 1109186 (ARIZ),

Loc. 51, 13 abril, 1942, E. O. Matthews s/n (ARIZ).

*Phellinus igniarius (L.) Quèl.

Ench. Fung. p. 177, 1886.

Material estudiado. Loc. 13, 17 abril, 1999, Cebrián Tovar 35

(ENCB).

Phellinus linteus (Berk. et Curt.) Teng

Fungi of China, p. 467, 1964

Material estudiado. Loc. 36, 12 diciembre, 1914, E. O.

Matthews s/n (ARIZ).

*Phellinus pini (Thore.: Fr.) Ames Ann. Mycol. 11:246, 1913

Material estudiado. Loc. 31, 20 abril, 1999, Cebrián Tovar 31 (ENCB).

Phellinus rimosus (Berk.) Pilát

Ann. Mycol. 38 (1): 80, 1940

Material estudiado. Loc. 36, 12 diciembre, 1915, E. O.

Matthews s/n (ARIZ), Loc. 51, 29 julio, 1974, J. Passini y M.F.

Robert 2051 (MEXU).

*Phellinus robineae (Murr.) Ames

Ann. Mycol. 11(3): 246, 1913

Material estudiado. Loc. 22, 20 diciembre, 1915, E. O.

Matthews 233981 (ARIZ).

Phellinus robustus (Karst.) Bourd. et Galz. (Fig. 4) Hym. France p. 616, 1928

Material estudiado. Loc. 6, 31 marzo, 1999, Moreno-Fuentes 508-B (FCME), 10 agosto, 1998, Díaz Moreno 575 (FCF-

UJED), 10 agosto, 1998, Díaz Moreno 564 (FCF-UJED), Loc.

11, 19 julio, 1992, Moreno-Fuentes 545 (FCME), 13 agosto,

1998, Moreno-Fuentes 530 (FCME), Loc. 20, 12 septiembre,

1998, Díaz Moreno 596 (FCF-UJED), Loc. 38, 20 abril, 1999,

Cebrián Tovar 9 (ENCB).

Phellinus tremulae (Bond.) Bond. et Boriss. (Fig. 5)

Polyporaceae Eur. USSR and Caucasia, p. 358, 1953

Material estudiado. Loc. 10, 9 agosto, 1992, Moreno-Fuentes

5714 (FCME). Loc. 14, 10 agosto, 2003, Díaz Moreno 732

(FCF-UJED). Loc. 40, 9 agosto, 1998, Díaz Moreno 554 (FCF-

UJED), Loc. 51, 8 septiembre, 1992, Moreno-Fuentes XVI 3

(FCME).

Phellinus weirianus (Bres.) Gilbn.

J. Ariz. Acad. Sci. 7: 137, 1972

Material estudiado. Loc. 6, 12 julio, 1992, Moreno-Fuentes 543 (FCME), Loc. 36, 2 mayo, 1915, E. O. Matthews 231567 (ARIZ), 4 diciembre, 1914, E. O. Matthews 231562 (ARIZ), 4 diciembre, 1914, E. O. Matthews 231585 (ARIZ), 16 agosto, 1915, E. O. Matthews 234310 (ARIZ), 2 mayo, 1914, E. O. Matthews 235275 (ARIZ), 12 diciembre, 1914, E. O. Matthews 235250 (ARIZ), 12 diciembre, 1914, E. O. Matthews s/n
(ARIZ), 12 diciembre, 1914, E. O. Matthews 235252 (ARIZ). Schizoporaceae

*Schizopora apacheriensis (Gilbn. et Canf.) Gilbn. et Ryv. N. Amer. Polyp., Vol. 2: 704, 1987

Material estudiado. Loc. 17, 29 septiembre, 1983, E. Pérez Silva 16408 (MEXU).

\section{Corticiaceae}

\section{Orden POLYPORALES}

Corticium alutaceum Pers.

Observ. Mycol. 1: 16, 1796

Material estudiado. Loc. 36, 2 diciembre, 1914, E. O. Matthews 283652 (ARIZ), 26 diciembre, 1914, E. O. Matthews 282347

(ARIZ).

Corticium roseum Pers.

Neves Mag. Bot. 1:111, 1794

Material estudiado. Loc. 36, 14 mayo, 1915, E. O. Matthews

283680 (ARIZ).

Corticium vellereum Ellis et Cragin

Bulletin of the Washburn Coll. Lab. Nat. Hist. 1: 66 ,

1885

Material estudiado. Loc. 32, 9 diciembre, 1914, E. O. Matthews

283747 (ARIZ).

Fomitopsidaceae

*Fomitopsis cajanderi (Karst.) Kotl. et Pouz. Ceska Mykol 11:157, 1957

Material estudiado. Loc. 40, 8 agosto, 1998, Díaz Moreno 555 (FCF-UJED).

*Fomitopsis palustris (B. et C.) Gilbn. et Ryv. Mycotaxon 22: 364, 1985

Material estudiado. Loc. 43, sin fecha, Laferrière 1109185 (ARIZ).

*Fomitopsis pinicola (Swartz.: Fr.) Karst.

Krit. Finl. Basidsv. p. 306, 1889

Material estudiado. Loc. 17, 11 agosto, 1998, Díaz Moreno 586 (FCF-UJED), Loc. 33, 20 abril, 1999, Cebrián Tovar 33

(ENCB).

Ganodermataceae

Ganoderma lucidum (Curtis) Karst.

Revue Mycol. Toulouse 3(9): 17,1881

Material estudiado. Loc. 6, 10 agosto, 1998, Díaz Moreno 565

(FCF-UJED). Loc. 43, 12 agosto, 1988, Laferrière 1109183

(ARIZ), Loc. 46, 8 julio, 1999, Díaz Moreno 538 (FCF-UJED).

*Ganoderma lobatum (Schw.) Atk. Ann. Mycol. 6:190. 1908

Material estudiado. Loc. 13, 9 agosto, 2003, Díaz Moreno 750 (FCF-UJED). Loc. 16, 20 abril, 1999, Cebrián Tovar 35

(ENCB).

Gloeophyllaceae

*Gloeophyllum carbonarium (Berk. et Curt.) Ryv. Mycotaxon 20: 334, 1984

Material estudiado. Loc. 41, 9 agosto, 2003, Díaz Moreno 744 (FCF-UJED).

Gloeophyllum mexicanum (Mont.) Ryv. Nord. J. Bot. 2: 79, 1982

Material estudiado. Loc. 40, 9 agosto, 1998, Díaz Moreno 557 (FCF-UJED). Loc. 50, 12 abril, 1915, E. O. Matthews 252867 (ARIZ), 15 diciembre, 1915, E. O. Matthews 252862 (ARIZ). Gloeophyllum sepiarium (Fr.) Karst. Finl. Httsv. 2:80, 1879

Material estudiado. Loc. 4, 19 octubre, 1973, P. Domínguez s/n (ENCB), Loc. 16, 11 agosto, 1998, Díaz Moreno 571 


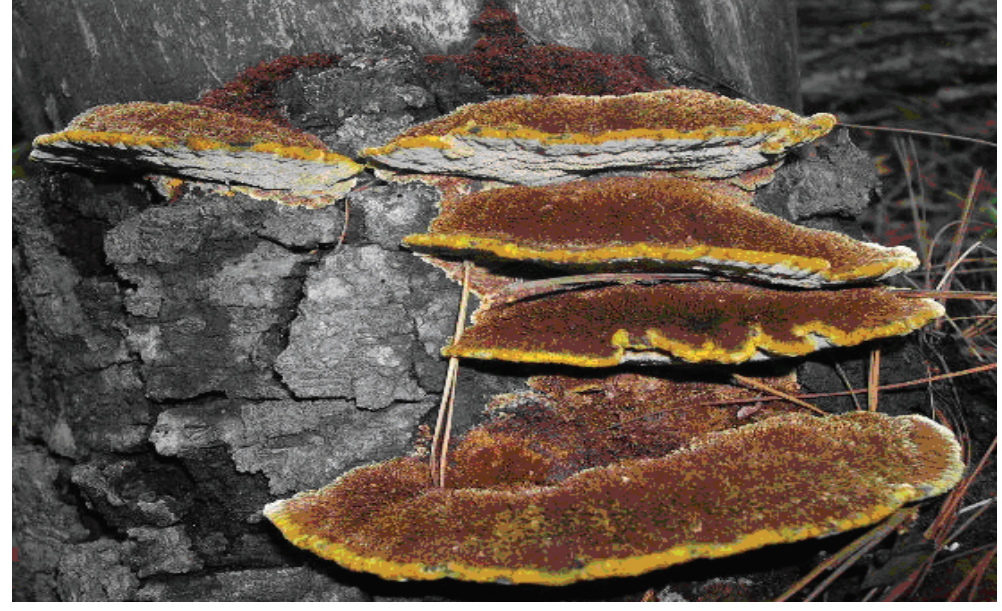

Figura 2. Inonotus fulvomelleus, x 0.5.

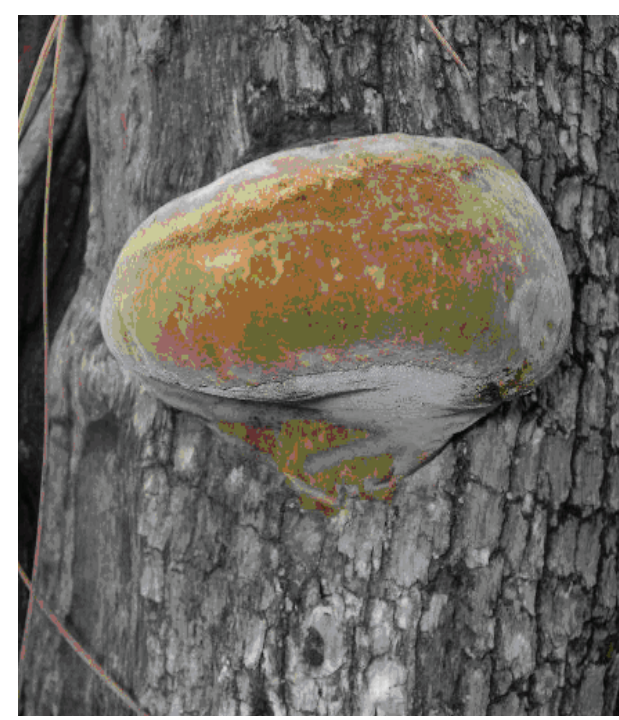

Figura 4. Phellinus robustus, x 0.2 .

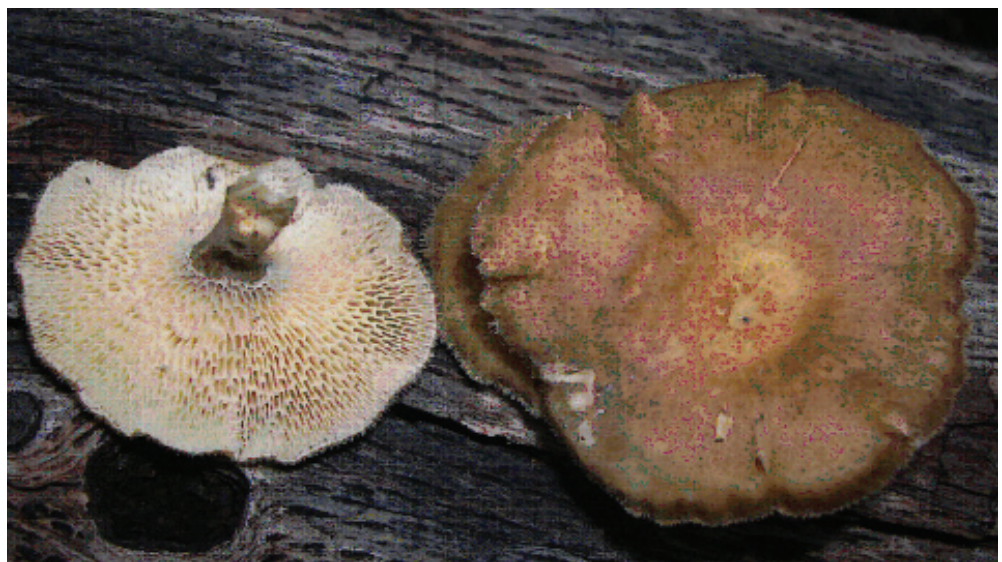

Figura 6. Polyporus arcularius, x 0.5 .

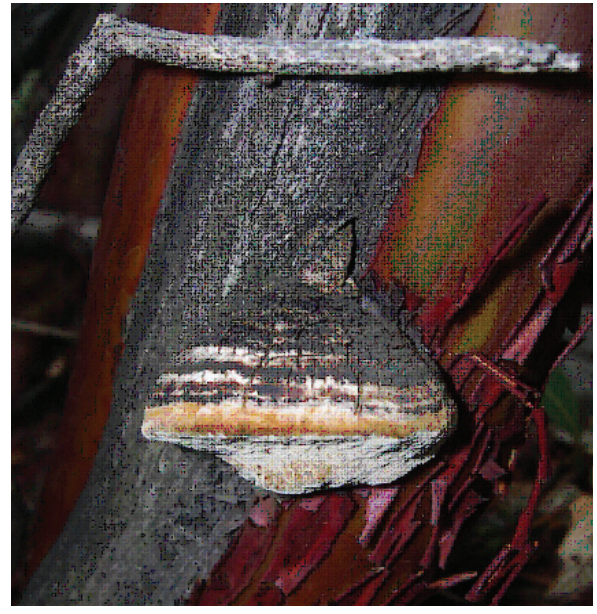

Figura 3. Phellinus arctostaphyli, x 1.

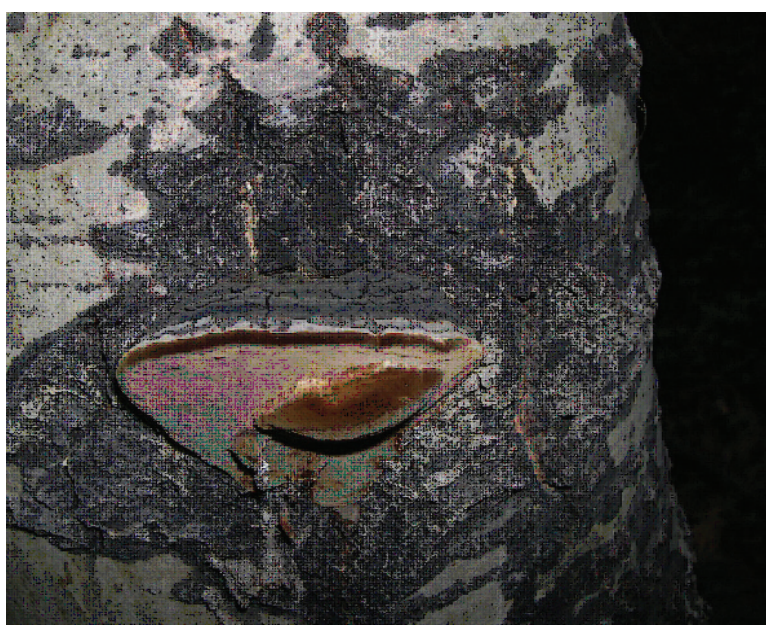

Figura 5. Phellinus tremulae, $\mathrm{x} 0.5$.

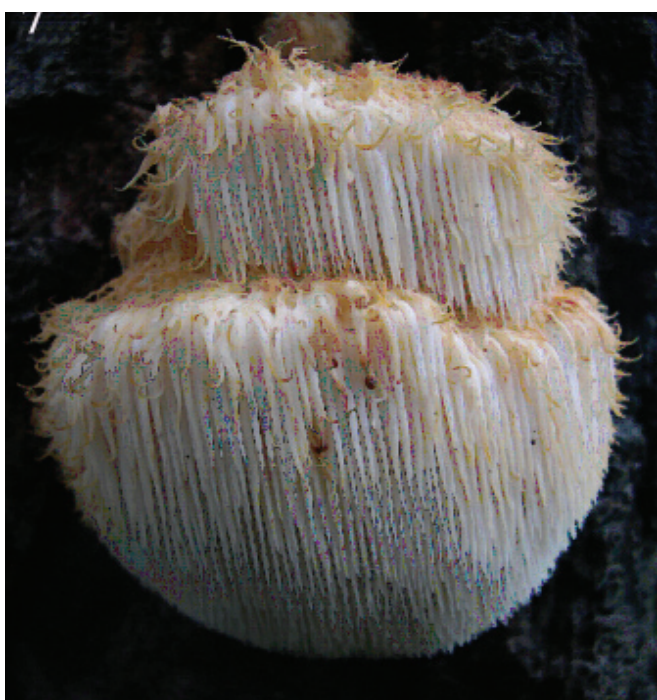

Figura 7. Hericium erinaceus, x 0.3 . 
(FCF-UJED), Loc. 17, 11 agosto, 1998, Díaz Moreno 579 (FCF-UJED), Loc. 21, 30 agosto, 1980, E. Pérez Silva 16306 (MEXU), Loc. 36, 12 agosto, 1915, E. O. Matthews s/n (ARIZ), E. O. 12 agosto, 1915, Matthews 251272 (ARIZ), Loc. 41, 9 septiembre, 1939, W J. Knobloch s/n (ARIZ), 9 septiembre, 1939, W. J. Knobloch 251393 (ARIZ), Loc. 43, 24 agosto, 1988, Laferrière 1109185 (ARIZ).

Gloeophyllum trabeum (Pers.) Murr. North American Flora 9 (2):129, 1908

Material estudiado. Loc. 36, 13 agosto, 1915, E. O. Matthews 252219 (ARIZ).

Meripilaceae

*Abortiporus biennis (Bull.) Sing. Mycologia 36:68, 1944

Material estudiado. Loc. 9, 30 julio, 1992, Moreno-Fuentes 5679 (FCME).

*Antrodia variiformis (Pk.) Donk Persoonia 4 (3): 340, 1966

Material estudiado. Loc. 27, 10 septiembre, 1009, Díaz Moreno 569(FCF-UJED).

Rigidoporus ulmarius (Sow.) Imaz. Bull. Gov. Forest. Exp. St. Tokio 57: 119, 1952

Material estudiado. Loc. 36, 12 diciembre, 1914, E. O. Matthews s/n (ARIZ).

Meruliaceae

*Byssomerulius corium (Pers.) Parm. Easti NSV Tead Akad. Toim. Biol. 16(4): 383, 1967 Material estudiado. Loc. 1, 12 agosto, 1942, E. O. Matthews s/n (ARIZ), 12 abril, 1942, E. O. Matthews s/n (ARIZ), Loc. 36, 4 abril, 1942, E. O. Matthews 256625 (ARIZ), 14 abril, 1942, E. O. Matthews 256356 (ARIZ).

*Byssomerulius incarnatus (Schw.) Gilbn. Fungi dec. Pond. Pine : 45, 1974

Material estudiado. Loc. 5, 11 agosto, 1998, Díaz Moreno 573 (FCF-UJED). Loc. 17, 11 agosto, 1998, Díaz Moreno 580 (FCF-UJED), Loc. 42, 27 septiembre, 1978, E. Pérez Silva 12694 (MEXU), 27 septiembre, 1978, E. Pérez Silva 12673 (MEXU), Loc. 43, 17 agosto, 1987, Laferrière 842 (ARIZ). *Merulius confluens Schw. Sch. Naturf. Ges. Leipzing 1: 92, 1822

Material estudiado. Loc. 1, 14 abril, 1922, E. O. Matthews 256356 (ARIZ), Loc. 36, 4 abril, 1942, E. O. Matthews 256625 (ARIZ), Loc. 39, 8 septiembre, 1998, Díaz Moreno 547 (FCFUJED).

\section{Polyporaceae}

Coriolopsis gallica (Fr.) Ryv. Norw. J. Bot. 19:230, 1973

Material estudiado. Loc. 15, 4 marzo, 1978, G. Guzmán s/n (ENCB), Loc. 36, 23 diciembre, 1914, E. O. Matthews 246460 (ARIZ), 12 diciembre, 1914, E. O. Matthews s/n (ARIZ), Loc. 47, 14 diciembre, 1914, Santreppe 246428 (ARIZ), 27 enero, 1915, E. O. Matthews 246422 (ARIZ).

Coriolopsis polyzona (Pers.) Ryv. Norw. J. Bot. 19: 230, 1972

Material estudiado. Loc. 22, 26 agosto, 1980, E. Pérez Silva 16392 (MEXU), Loc. 27, 27 agosto 1980, E. Pérez Silva 16430 (MEXU), Loc. sin precisar, diciembre, 1891, Hartmann 213926 (ARIZ).

*Cryptoporus volvatus (Peck) Shear Bull. Torrey Bot. Club 29: 450, 1902 Material estudiado. Loc. 13, 9 agosto, 2003, Díaz Moreno 751 (FCF-UJED).
Datronia mollis (Smf.) Donk

Persoonia 4 (3):338, 1966

Material estudiado. Loc. 19, sin fecha, E. O. Matthews NP-

3126 (ARIZ).

Fomes fasciatus (Sw.: Fr.) Cke. Grevillea 14: 21, 1885

Material estudiado. Loc. 2, 6 septiembre, 1915, E. O. Matthews 271400 (ARIZ), 16 agosto, 1915, E. O. Matthews 234310 (ARIZ), Loc. 36, 12 agosto, 1915, E. O. Matthews s/n (ARIZ), 16 agosto, 1915, E. O. Matthews 271390 (ARIZ).

Lenzites betulina (Fr.) Fr. Epicr. Syst. Mycol. p. 405, 1838

Material estudiado. Loc. 8, 26 septiembre, Díaz Moreno 509 (FCF-UJED), 23 septiembre 1992, Moreno-Fuentes 5627 (FCME), Loc. 25, 29 agosto, 1980, Pérez Silva 19869 (MEXU), Loc. 43, 25 agosto, 1987, Laferrière 1109184 (ARIZ).

*Oligoporus caesius (Schrad.: Fr.) Gilbn. et Ryv. Mycotaxon 22:365, 1985

Material estudiado. Loc. 17, 11 septiembre, 1998, Díaz Moreno 587, Loc. 20, 11 septiembre, 1998, Díaz Moreno 577 (FCFUJED).

*Oligoporus fragilis (Fr.) Gilbn et Ryv. Mycotaxon 22:365,1985

Material estudiado. Loc. 6, 10 agosto, 1998, Díaz Moreno 569 (FCF-UJED).

*Pachykytospora papyracea (Schwein.) Ryv. Norw. Jl. Bot.19:233, 1972

Material estudiado. Loc. 18, 12 septiembre, 1998, Díaz Moreno 559, Loc. 39, 8 septiembre, 1998, Díaz Moreno 545 (FCFUJED).

*Perenniporia narymica (Pil.) Pouz.

Ceska. Mykol. 38: 204, 1984

Material estudiado. Loc. 13, 9 agosto, 2003, Díaz Moreno 735 (FCF-UJED), Loc. 39, 7 septiembre, 1998, Díaz Moreno 542 (FCF-UJED), 8 septiembre, 1998, Díaz Moreno 546 (FCFUJED).

Phaeolus schweinitzii (Fr.) Pat.

Ess. Taxon. Hym. p. 86, 1900

Material estudiado. Loc. 6, 1 agosto, 1997, Moreno-Fuentes 528 (FCME), Loc. 8, 13 agosto, 1992, Moreno-Fuentes 571 (FCME), 15 septiembre, 1992, Moreno-Fuentes 5719 (FCME), Loc. 13, 9 agosto, 2003, Díaz Moreno 742 (FCF-UJED), 9 agosto, 2003, Díaz Moreno 748 (FCF-UJED), Loc. 43, 8 agosto, 1988, Laferrière 1109190 (ARIZ).

Polyporus arcularius (Batsch.) Fr. (Fig. 6) Syst. Mycol. 1: 342, 1821

Material estudiado. Loc. 36, 12 diciembre, 1914, E. O.

Matthews s/n (ARIZ), Loc. 41, 30 junio, 1939, Knobloch s/n (ARIZ), 30 junio, 1939, Knobloch 203420 (ARIZ).

Polyporus squamosus (Huds.) Fr. Syst. Mycol. 1:343. 1821

Material estudiado. Loc. 6, 10 agosto, 1998, Díaz Moreno 563 (FCF-UJED).

Polyporus alveolaris Lafer. et Gilbn.

Mycotaxon 37:331. 1990

Material estudiado. Loc. 43, sin fecha, Laferrière 602 (ARIZ). 60. Polyporus tricholoma Mont. Ann. Sci. Nat. 8: 365,1837

Material estudiado. Loc. 13, 9 agosto, 2003, Díaz Moreno 733 (FCF-UJED), Loc. 24, 28 agosto, 1980, E. Pérez Silva 16490 (MEXU), Loc. 39, 8 julio, 1998, Díaz Moreno 540 (FCF- 
UJED), Loc. 44, 28 septiembre, 1978, E. Pérez Silva 12488 (MEXU).

*Polyporus varius (Pers.) Fr. Syst. Mycol. 1:352, 1821

Material estudiado. Loc. 5, 9 julio, 1998, Díaz Moreno 561 (FCF-UJED), Loc. 40, 8 agosto, 1998, Díaz Moreno 556 (FCFUJED), 8 septiembre, 1998, Díaz Moreno 556 (FCF-UJED), 8 septiembre, 1998, Díaz Moreno 561 (FCF-UJED).

Pycnoporus cinnabarinus (Jacq.) Fr.

Rev. Mycol. 3: 18, 1881

Material estudiado. Loc. 43, 6 mayo, 1988, Laferrière 1109196 (ARIZ).

Pycnoporus sanguineus (L.) Murr.

Bull. Torrey Bot. Club 3: 421, 1904

Material estudiado. Loc. 2, 19 octubre, 1973, P. Domínguez s/n

(ENCB).

*Pyrofomes demidoffi (Lèv.) Kotl. et Pouz.

Voy. Russ. Merid. 2: 92, 1842

Material estudiado. Loc. 26, 27 agosto, 1980, Pérez Silva

16408 (MEXU), Loc. 29, 31 julio, 1998, Moreno-Fuentes 450

(FCME).

Trametes cervina (Schw.) Bres.

Ann. Mycol. 1:81, 1903

Material estudiado. Loc. 17, 11 agosto, 1998, Díaz Moreno

582 (FCF-UJED), Loc. 25, 29 agosto, 1980, E. Pérez Silva

19901 (MEXU), Loc. 39, 8 julio, 1998, Díaz Moreno 550 (FCFUJED).

Trametes hirsuta (Wulf.) Pilát

Atl. Champ. Europ. 3:265, 1939

Material estudiado. Loc. 6, sin fecha, Hartmann s/n (ARIZ).

Loc. 36, 12 diciembre, 1914, E. O. Matthews s/n (ARIZ). Sin

localidad, 13 diciembre, 1914, Santreppe 246428 (ARIZ).

Trametes membranacea (Sw.) Kreisel

Ciencias Biol. Univ. Habana, Ser. 4,16: 83, 1971

Material estudiado. Loc. 43, 30 julio, 1987, Laferrière 1107469

(ARIZ).

Trametes versicolor (L.:Fr.) Pilát

Atl. Champ. Eur. 3:261, 1936

Material estudiado. Loc. 4, 3 mayo, 1981, P. Domínguez s/n

(ENCB), Loc. 6, 10 agosto, 1998, Díaz Moreno 566 (FCF-

UJED), Díaz Moreno 558 (FCF-UJED), Loc. 10, 9 agosto,

1992, Moreno-Fuentes 5724 (FCME), Loc. 11, 2 junio, 1915,

E. O. Matthews 255011 (ARIZ), Loc. 20, 31 agosto, 1980, E.

Pérez Silva 17186 (MEXU), Loc. 21, 13 abril, 1980, Jorge

Caballero 13102 (ENCB), Loc. Sin precisar, 12 septiembre,

1960, F. Sánchez Pereyra 3710 (ENCB).

Trametes villosa (Fr.) Kreisel

Ciencias Biol. Univ. Habana, Ser. 4,16: 84, 1971

Material estudiado. Loc. 9, 18 septiembre, 1992, Moreno-

Fuentes 5651 (FCME), Loc. 16, 11 agosto, 1998, Díaz Moreno

572 (FCF-UJED).

Trichaptum abietinum (Dicks.) Ryv.

Norw. J. Bot. 19:237, 1972

Material estudiado. Loc. 16, 11 agosto, 1998, Díaz Moreno 576 (FCF-UJED), 12 agosto, 1998, Díaz Moreno 594 (FCF-UJED), Loc. 17, 11 agosto, 1998, Díaz Moreno 581 (FCF-UJED), Loc. 18, 12 agosto, 1998, Díaz Moreno 588 (FCF-UJED), Loc. 39, 8 julio, 1998, Díaz Moreno 541 (FCF-UJED), Loc. 40, 8 septiembre, 1998, Díaz Moreno 560 (FCF-UJED)

Trichaptum biforme (Fr.) Ryv.

Norw. J. Bot. 19:237, 1972
Material estudiado. Loc. 5, 9 agosto, 1998, Díaz Moreno 562 (FCF-UJED). Loc. 12, 1 agosto, 1992, Moreno-Fuentes 5672 (FCME), Loc. 13, 9 agosto, 2003, Díaz Moreno 736 (FCFUJED), 9 agosto, 2003, Díaz Moreno 746 (FCF-UJED), Loc. 39, 8 agosto, 1998, Díaz Moreno 549 (FCF-UJED).

Steccherinaceae

Antrodiella incrustans (B. et C. ex Sacc.) Ryv. Mycotaxon 18: 344, 1984

Material estudiado. Loc. 36, 4 diciembre, 1914, E. O. Matthews s/n (ARIZ).

Bondarsewiaceae

*Amylosporus campbelli (Berk.) Ryv. Norw. J. Bot. 24: 217, 1977

Material estudiado. Loc. 36, sin fecha, E. O. Matthews 200133 (ARIZ).

*Bondarsewia berkeleyi (Fr.) Bond. et Sing. Ann Mycol. 39:47, 1941

Material estudiado. Loc. 50, 15 diciembre, 1915, E. O.

Matthews s/n (ARIZ).

Hericiaceae

*Hericium erinaceus (Bull.) Pers. (Fig. 7) Comment. Fungus Clavaeform: 27, 1797

Material estudiado. Loc. 2, 19 agosto, 1982, E. Pérez Silva 18698 (MEXU), Loc.20 , 31 agosto, 1980, M. A. Martínez 17028 (MEXU), Loc. 23, 27 agosto, 1980, E. Pérez Silva 16411 (MEXU), Loc. 30, 8 agosto, 1997, Moreno-Fuentes 360 (FCME), Loc. 31, 20 julio, 1997, Moreno-Fuentes 538 (FCME).

Stereaceae

\section{Orden RUSSULALES}

Stereum complicatum (Fr.) Fr. Epicr. Syst. Mycol. p. 548, 1838

Material estudiado. Loc. 20, 31 agosto, 1980, M. A. Martínez 17025 (MEXU).

Stereum fasciatum (Schw.) Fr. Epicr. Syst. Mycol. p. 546, 1836

Material estudiado. Loc. 20, 31 agosto, 1980, E. Pérez Silva 16281 (MEXU), Loc. 36, 6 septiembre, 1915, E. O. Matthews 271400 (ARIZ), 13 marzo, 1916, E. O. Matthews 271390 (ARIZ).

Stereum gausapatum (Fr.) Fr. Hym. Eur. p. 638, 1874

Material estudiado. Loc. 24, 28 agosto, 1980, E. Pérez Silva 16487 (MEXU), Loc. 38, 4 abril, 1978, G. Guzmán 16911 (ENCB), Loc. 42, 27 septiembre, 1978, E. Pérez Silva 12673(MEXU), 27 septiembre, 1978, E. Pérez Silva 12660 (MEXU).

Stereum heterosporum Burt

Ann. Mo. Bot. Gdn. 7: 220, 1920

Material estudiado. Loc. 36, 27 diciembre, 1914, E. O. Matthews 272459 (ARIZ), 11 mayo, 1915, E. O. Matthews 272469 (ARIZ), Loc. 47, 27 enero, 1942, E. O. Matthews s/n (ARIZ).

Stereum hirsutum (Willd.) Pers. Observ. Mycol. 2: 90, 1800

Material estudiado. Loc. 13, 9 agosto, 2003, Díaz Moreno 747 (FCF-UJED), Loc. 20, 23 febrero, 1981, J. García 1133 (ENCB), 31 agosto, 1980, M. A. Martínez, 17025 (MEXU), Loc. 21, 23 febrero, J. García 2035 (ENCB), 25 febrero, 1981, J. García 1129 (ENCB), Loc. 27, 27 agosto, 1980, E. Pérez Silva 16442 (MEXU), 27 agosto, 1980, E. Pérez Silva 12694 
(MEXU), Loc. 42, 27 septiembre, 1978, E. Pérez Silva 12658 (MEXU), 31 agosto, 1980, Ana María Ruiz 16280 (MEXU), Loc. 43, 27 agosto, 1988, Laferrière 1109181 (ARIZ), 24 agosto, 1988, Laferrière 1109178 (ARIZ).

Stereum ochraceoflavum (Schw.) Fr. N. Am. Fungi, p. 17, 1878

Material estudiado. Loc. 43, 22 agosto, 1988, Laferrière 1725 (ARIZ), 27 agosto, 1988, Laferrière 1822 (ARIZ), 22 agosto, 1988, Laferrière 1736 (ARIZ).

Stereum ostrea (Blume et T. Ness.) Fr. Epicr. Syst. Mycol. p. 574, 1838

Material estudiado. Loc. 13, 9 agosto, 2003, Díaz Moreno 734 (FCF-UJED), Loc. 17, 7 septiembre, 1998, Díaz Moreno 539 (FCF-UJED), 11 septiembre, 1998, Díaz Moreno 199 (FCF-
UJED), Loc. 18, 12 septiembre, 1998, Díaz Moreno 591 (FCFUJED), Loc. 20, 11 septiembre. 1998, Díaz Moreno 574 (FCFUJED), Loc. 32, 20 abril, 1999, Cebrián Tovar 31-B (ENCB). Loc. 33, 20 abril, 1999, Cebrián Tovar 31-b (ENCB), Loc. 35, 25 febrero, 1981, J. García s/n (ENCB), Loc. 39, 8 septiembre, 1998, Díaz Moreno 543, Loc. 40, 8 septiembre, 1998, Díaz Moreno 559 (FCF-UJED), Loc. 46, 3 septiembre, 1972, Huerta $\mathrm{s} / \mathrm{n}(\mathrm{ENCB})$.

\section{Bankeraceae}

\section{Orden THELEPHORALES}

Boletopsis subsquamosa (Fr.) Kotl. et Pouz. Ceska Mykol. 11 (3):164, 1957

Material estudiado. Loc. 16, 30 agosto, 1980, Pérez Silva s/n (MEXU).

Apéndice 2. Lista de las localidades estudiadas por municipio en el estado de Chihuahua.

\section{Camargo}

1. Santa Bárbara, $103^{\circ} 58^{\prime} 09^{\prime \prime}-28^{\circ} 18^{\prime} 16^{\prime \prime}, 1288 \mathrm{~m} \mathrm{snm}$

\section{Chihuahua}

2. El Rosario, $106^{\circ} 28^{\prime} 24^{\prime \prime}-30^{\circ} 14^{\prime} 05^{\prime \prime}, 1360 \mathrm{~m} \mathrm{snm}$.

3. San Isidro, $106^{\circ} 19^{\prime} 43^{\prime \prime}-30^{\circ} 51^{\prime} 08^{\prime \prime}, 1230 \mathrm{~m} \mathrm{snm}$.

\section{San Buena Ventura}

4. Cañada de Chilladeros, Sierra del Nido. Carretera ChihuahuaCd. Juárez, Km 40, al NO del entronque a Santa Clara. $106^{\circ}$ $54^{\prime} 43^{\prime \prime}-29^{\prime} 56^{\prime} 44^{\prime \prime}, 1617 \mathrm{~m} \mathrm{snm}$.

\section{Bocoyna}

5. El cuervo, cerca de El Ranchito, próximo a la brecha a San Juanito. $107^{\circ} 12^{\prime} 36^{\prime \prime}-26^{\circ} 57^{\prime} 14^{\prime \prime}, 2420$ m snm.

6. Alrededores de Garajewachi, Panalachi, $107^{\circ} 20^{\prime} 59.5^{\prime \prime}-27^{\circ}$ 42'25.4", $2200 \mathrm{~m} \mathrm{snm}$.

7. La Laguna, $\mathrm{km} 5$ al E de Bocoyna, $107^{\circ} 13^{\prime} 20^{\prime \prime}-27^{\circ}$ $08^{\prime} 13^{\prime \prime}, 2300 \mathrm{~m} \mathrm{snm}$.

8. Huirinia, $5 \mathrm{~km}$ al E de San Juanito, $107^{\circ} 18^{\prime} 22^{\prime \prime}-27^{\circ}$ 46’34", $2400 \mathrm{~m} \mathrm{snm}$.

9. Bahuirachi, $6 \mathrm{~km}$ al SO de Panalachi, $107^{\circ} 34^{\prime} 13^{\prime \prime}-27^{\circ}$

$40^{\prime} 21^{\prime \prime}, 2250 \mathrm{~m} \mathrm{snm}$.

10. Morá a $6 \mathrm{~km}$ al NO de San Juanito, $107^{\circ} 36^{\prime} 01^{\prime \prime}-27^{\circ}$

$58^{\prime} 12^{\prime \prime}, 2400 \mathrm{~m} \mathrm{snm}$.

11. Carretera Garewachi Panalachi, $107^{\circ} 45^{\prime} 44^{\prime \prime}-27^{\circ} 57^{\prime} 01^{\prime \prime}$, $2360 \mathrm{~m} \mathrm{snm}$.

12. Mesa del Oso, $10 \mathrm{~km}$ al NO de $107^{\circ} 46^{\prime} 24^{\prime \prime}-27^{\circ} 58^{\prime} 16^{\prime \prime}$, $2400 \mathrm{~m} \mathrm{snm}$.

13. San Juanito a $20 \mathrm{~km}$ al $\mathrm{N}$ de la Carretera Gran Visión, rumbo a Basaseachi, $107^{\circ} 37^{\prime} 01^{\prime \prime}$ - $27^{\circ} 58^{\prime} 12^{\prime \prime}, 2400 \mathrm{~m} \mathrm{snm}$.

\section{Cuauhtémoc}

14. Valle de Allende. $106^{\circ} 53^{\prime} 09^{\prime \prime}-28^{\circ} 50^{\prime} 32^{\prime \prime}, 2100 \mathrm{~m} \mathrm{snm}$. 15. Anáhuac, $106^{\circ} 44^{\prime} 39^{\prime \prime}-28^{\circ} 28^{\prime} 48^{\prime \prime}, 1905$ m snm.

\section{Guachochi}

16. Km. 78, carretera Creel-Guachochi, $107^{\circ} 18^{\prime} 49.8^{\prime \prime}-27^{\circ}$ $16^{\prime} 42.3^{\prime \prime}, 2000 \mathrm{~m}$ snm.

17. Entronque a Cibarachic, $\mathrm{km} \mathrm{19+800,} \mathrm{brecha} \mathrm{Cerro} \mathrm{Grande} \mathrm{a}$ Tonachic, $107^{\circ} 02^{\prime} 50^{\prime \prime}-27^{\circ} 12^{\prime} 37^{\prime \prime} 2122 \mathrm{~m} \mathrm{snm}$.

18. Km. 111, alrededores de Laguna de Cabórachi, carr. Creel-

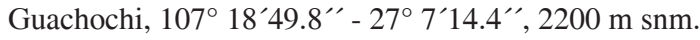
19. Cañón de la Sinforosa, $107^{\circ} 05^{\prime} 09^{\prime \prime}-26^{\circ} 42^{\prime} 23^{\prime \prime}, 2400$ $\mathrm{m}$ snm.

20. Km. 71, carretera Creel-Guachochi, a $1 \mathrm{~km}$ del Puente de Urique, $107^{\circ} 30^{\prime} 06^{\prime \prime}-27^{\circ} 35^{\prime} 16^{\prime \prime}, 2400 \mathrm{~m} \mathrm{snm}$.

21. La Laja, km 82, carretera Creel-Guachochi, $107^{\circ} 31^{\prime} 16^{\prime \prime}$ $27^{\circ} 34^{\prime} 12^{\prime \prime}, 2300 \mathrm{~m} \mathrm{snm}$.

22. Cabórachi, $107^{\circ} 26^{\prime} 53^{\prime \prime}-27^{\circ} 33^{\prime} 57^{\prime \prime}, 2420 \mathrm{~m} \mathrm{snm}$.

23. Km. 32 O de la carretera Guachochi-Balleza, $107^{\circ} 35^{\prime} 12^{\prime \prime}$ $27^{\circ} 37^{\prime} 16^{\prime \prime}, 2300 \mathrm{~m} \mathrm{snm}$.

24. Rincón del Manzano, al O de Cabórachi, $106^{\circ} 56^{\prime 2} 20^{\prime \prime}-26^{\circ}$ $42^{\prime 2} 29^{\prime \prime}, 2340 \mathrm{~m} \mathrm{snm}$.

25. Km. 10 carretera Guachochi-Balleza. $107^{\circ} 07^{\prime} 30^{\prime \prime}-26^{\circ} 49^{\prime \prime}$ $10^{\prime \prime}, 1160 \mathrm{~m} \mathrm{snm}$.

26. Km. 28 carretera Guachochi-Ballleza, $107^{\circ} 35^{\prime} 34^{\prime \prime}-27^{\circ}$

$37^{\prime} 16^{\prime \prime}, 2300 \mathrm{~m} \mathrm{snm}$

27. Km. 52 carretera Guachochi-Balleza, Mesa Agostadero de los Aguirre. $106^{\circ} 45^{\prime} 41^{\prime \prime}-26^{\circ} 54^{\prime} 49^{\prime \prime}, 2340 \mathrm{~m} \mathrm{snm}$.

28. Rancho las Adjuntas, $107^{\circ} 07^{\prime} 30^{\prime \prime}-27^{\circ} 37^{\prime} 33^{\prime \prime}, 2040$ $\mathrm{m}$ snm.

29. Carretera Tonachi-Sawararé, $107^{\circ} 33^{\prime} 35^{\prime \prime}-27^{\circ} 20^{\prime} 06^{\prime \prime}$, $2320 \mathrm{~m} \mathrm{snm}$.

30. Rancho La Esperanza, $107^{\circ} 11^{\prime} 42^{\prime \prime}-27^{\circ} 06^{\prime} 25^{\prime \prime}, 2400$ $\mathrm{m}$ snm.

31. Camino Matachic-Tonachi, $107^{\circ} 16^{\prime} 36^{\prime \prime}-26^{\circ} 56^{\prime} 47^{\prime \prime}, 2020$ $\mathrm{m}$ snm.

32. Arroyo de Cajirachi, ejido de Santa Anita, $107^{\circ} 26^{\prime} 32^{\prime \prime}$ $26^{\circ} 50^{\prime} 05^{\prime \prime}, 2200 \mathrm{~m} \mathrm{snm}$.

33. Arroyo de Remochi, ejido de Tonachi, $107^{\circ} 06^{\prime} 13^{\prime \prime}-27^{\circ}$

$10^{\prime} 18^{\prime \prime}, 2150 \mathrm{~m} \mathrm{snm}$.

34. El Guajolote, ejido de Santa Anita, $107^{\circ} 19^{\prime} 52^{\prime \prime}-26^{\circ}$

$50^{\prime} 44^{\prime \prime}, 2580 \mathrm{~m} \mathrm{snm}$.

35. Carretera Creel-Guachochi, $80 \mathrm{~km}$ al S de Creel, desviación a Batopilas, $107^{\circ} 30^{\prime} 12^{\prime \prime}-27^{\circ} 33^{\prime} 06^{\prime \prime}, 2300 \mathrm{~m} \mathrm{snm}$.

\section{Hidalgo Del Parral}

36. Parral, $105^{\circ} 39^{\prime} 47^{\prime \prime}-26^{\circ} 55^{\prime} 57^{\prime \prime}, 1720 \mathrm{~m} \mathrm{snm}$.

\section{Jiménez}

37. Norte de Jiménez, Río San Isidro, $104^{\circ} 55^{\prime 2} 24^{\prime \prime}-27^{\circ}$

$07^{\prime} 48^{\prime \prime}, 1262 \mathrm{~m} \mathrm{snm}$. 


\section{Maderas}

38. Bajada a Piedras Verdes, ejido El Lago, $108^{\circ} 34^{\prime} 06^{\prime \prime}-28^{\circ}$ 43'59', $1800 \mathrm{~m} \mathrm{snm}$.

\section{Ocampo}

39. Bajada de la caída de agua a La Ventana, Cascada de

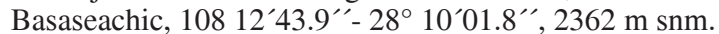

40. Camino de terracería de Basaseachic a San Juanito, km 54, $107^{\circ} 56^{\prime} 38.3^{\prime \prime}-28^{\circ} 6^{\prime} 25.5^{\prime \prime}, 2320 \mathrm{~m} \mathrm{snm}$.

41. Mojarachic, $108^{\circ} 06^{\prime} 39^{\prime \prime}-28^{\circ} 06^{\prime} 13^{\prime \prime}, 2180 \mathrm{~m} \mathrm{snm}$.

\section{Temosachi}

42. Piedra Volada, $108^{\circ} 22^{\prime} 15^{\prime \prime}-28^{\circ} 21^{\prime} 15^{\prime \prime}, 1740 \mathrm{~m} \mathrm{snm}$.

43. Nabogame, $108^{\circ} 28^{\prime} 50^{\prime \prime}-28^{\circ} 29^{\prime} 43^{\prime \prime}, 1640 \mathrm{~m} \mathrm{snm}$.

44. Presa estación $108^{\circ} 22^{\prime} 15^{\prime \prime}-28^{\circ} 21^{\prime} 15^{\prime \prime}, 1780 \mathrm{~m} \mathrm{snm}$.

45. Km. 259 carretera Chihuahua-Basaseachic, comunidad El
Pajarito $108^{\circ} 06^{\prime} 39^{\prime \prime}-28^{\circ} 06^{\prime} 13^{\prime \prime}, 2180 \mathrm{~m} \mathrm{snm}$.

\section{Riva Palacio}

46. Km. 28 de la brecha del Parque Cumbres de Majalca, $106^{\circ}$ $27^{\prime} 27.5^{\prime \prime}-28^{\circ} 47^{\prime} 45.5^{\prime \prime}, 2046 \mathrm{~m} \mathrm{snm}$.

47. Río Florido, $105^{\circ} 02^{\prime} 20^{\prime \prime}$ - $27^{\circ} 41^{\prime} 13^{\prime \prime}, 1260 \mathrm{~m} \mathrm{snm}$.

\section{Urique}

48. Urique, rumbo a La Bufa, $109^{\circ} 51^{\prime} 00^{\prime \prime}-27^{\circ} 09^{\prime} 05^{\prime \prime}, 560$ $\mathrm{m}$ snm.

49. Cascada de Cerocahui, $108^{\circ} 03^{\prime} 20^{\prime \prime}-27^{\circ} 17^{\prime} 53^{\prime \prime}, 1620 \mathrm{~m}$ snm.

50. Barjas. $107^{\circ} 54^{\prime} 58^{\prime \prime}-27^{\circ} 05^{\prime} 58^{\prime \prime}, 2060 \mathrm{~m} \mathrm{snm}$.

Valle de Zaragoza

51. Cañón de Zaragoza. $105^{\circ} 48^{\prime} 39^{\prime \prime}-27^{\circ}$ 27’26”, 1340 m snm. 